\title{
Effet Du Fumier Sur Le Bilan Des Éléments Nutritifs Des Champs Dans Le Terroir De La Néma Au Saloum (Sénégal)
}

\author{
Ismaila Coly, PhD \\ Université Assane SECK de Ziguinchor, UFR Sciences et Technologies, \\ Département d'Agroforesterie, Laboratoire d'Agroforesterie et d'Ecologie, \\ Ziguinchor, Sénégal \\ Babacar Diop, PhD
}

Université Cheikh Anta DIOP de Dakar, Faculté des Sciences et Techniques, Département de Biologie Végétale, Dakar, Sénégal

\section{Arfang Ousmane Kémo Goudiaby}

Université Assane SECK de Ziguincho, UFR Sciences et Technologies, Département d'Agroforesterie, Laboratoire d'Agroforesterie et d'Ecologie, Ziguinchor, Sénégal

Doi: 10.19044/esj.2018.v14n27p126 URL:http://dx.doi.org/10.19044/esj.2018.v14n27p126

\begin{abstract}
In the land of Nema, fields show low agricultural potential due to high human and climatic pressure. To increase their agricultural production, the populations have opted either for the use of chemical fertilizer or for the supply of manure through the Agriculture-Livestock integration. This paper focuses on evaluating the effect of on-farm use of manure on groundnut pod and groundnut production, and nutrient balance at the field level. 20 square plots of $2 \mathrm{~m}$ each were used per field on the monitored farms. The hay and pod samples were taken therein, weighed, and dried in an oven at $60^{\circ} \mathrm{C}$ to a constant weight. This work was carried out for both fertilized fields and unfertilized fields (controls). In these same farms, samples of manure produced were collected and analyzed. The production of peanut and groundnut pods in the fertilized fields and in the control fields shows an overall positive effect of the manure on the yields in the land of the Néma. Taking into account the figures of the average deficits, the nitrogen, phosphorus, and potassium balance is deficient in ground-level groundnut peanut fields. This is due to the low manure doses per hectare and its low nutrient content.
\end{abstract}

Keywords: Land of Nema, nutrients, manure, hay, pods 


\section{Résumé}

Dans le terroir de la Néma, les terres montrent de faibles potentiels agricoles du fait de la forte pression anthropique et climatique. Ainsi, pour accroître leur production agricole, les populations ont opté soit pour l'utilisation de l'engrais chimique soit pour l'apport de fumier à travers l'intégration Agriculture-Elevage. Cette étude vise à évaluer l'effet de l'utilisation du fumier produit dans l'exploitation agricole sur la production de gousses et de fane d'arachide et le bilan des éléments nutritifs au niveau des champs. Ainsi, 20 placettes carrées de $2 \mathrm{~m}$ de côté ont été utilisées par champ dans les exploitations agricoles suivies. Les échantillons de fanes et de gousses sont prélevés dans ces dernières, pesés et séchés à l'étuve à $60^{\circ} \mathrm{C}$ jusqu'à poids constant. Ce travail est réalisé aussi bien pour les champs fumés que pour les champs non fertilisés (témoins). Dans ces mêmes exploitations agricoles, des échantillons de fumier produit ont été prélevés et analysés. La production de fane et de gousses d'arachide dans les champs fumés et dans les champs témoins fait apparaître globalement dans le terroir de la Néma un effet positif $\mathrm{du}$ fumier sur les rendements. Tenant en compte les chiffres des déficits moyens, le bilan en azote, phosphore et potassium est déficitaire dans les champs d'arachide fumés à l'échelle du terroir du fait de la faiblesse des doses de fumier apportées à l'ha et de sa faible teneur en éléments nutritifs.

Mots clés: Terroir de la Néma, éléments nutritifs, fumier, fane, gousses

\section{Introduction}

Dans la zone soudano-sahélienne, le rapide accroissement démographique, et ainsi les besoins importants à satisfaire en produits alimentaires ont entraîné une forte pression foncière (Kleene et al., 1989 ; CMDT, 1993; Kaasschieter \& Coulibaly, 1995; Akpo et al., 2004). Avec l'augmentation des superficies cultivées, les terres cultivables sont devenues rares. La disparition des temps de jachère (Ngom, 2000 ; Akpo et al., 2002, 2003) a entraîné une raréfaction des terres de parcours, car dans le terroir de la Néma même les zones marginales, autrefois réservées aux animaux, sont désormais intégrées dans les systèmes de culture continue. Les terres continuellement cultivées ont fini par montrer alors de faibles potentiels agricoles (Borderon, 1989 ; Ndao, 2001).

La fertilisation étant un moyen important d'obtenir de hauts rendements (Debreczeni et Berecz, cités par Bossissi, 2010), les populations ont désormais opté pour une intensification de l'agriculture. Celle-ci passe soit par une utilisation des engrais minéraux, essentiellement pour les cultures de rente (arachide), soit par une gestion intégrée des ressources végétales et animales. L'intégration Agriculture - Elevage vise à améliorer la production 
agricole tout en préservant la fertilité des terres par le fumier de ferme. Les apports d'éléments fertilisants se font à travers l'épandage de fumier. Ce dernier est obtenu par recyclage des résidus de récolte (fane d'arachide essentiellement) utilisés pour le bétail (Madjikam, 2000).

Le présent travail a étudié l'utilisation du fumier dans un champ de production de gousses et de fanes d'arachide et le bilan des éléments nutritifs dans les exploitations.

\section{Matériel et Méthodes}

\section{La zone d'étude}

D'une superficie de $50 \mathrm{~km}^{2}$ (Lienou, 1995), le bassin versant de la Néma est compris entre $13^{\circ} 42^{\prime}$ à $13^{\circ} 45^{\prime}$ 'Nord et $16^{\circ} 22^{\prime}$ à $16^{\circ} 29^{\prime}$ Ouest (Figure 1).

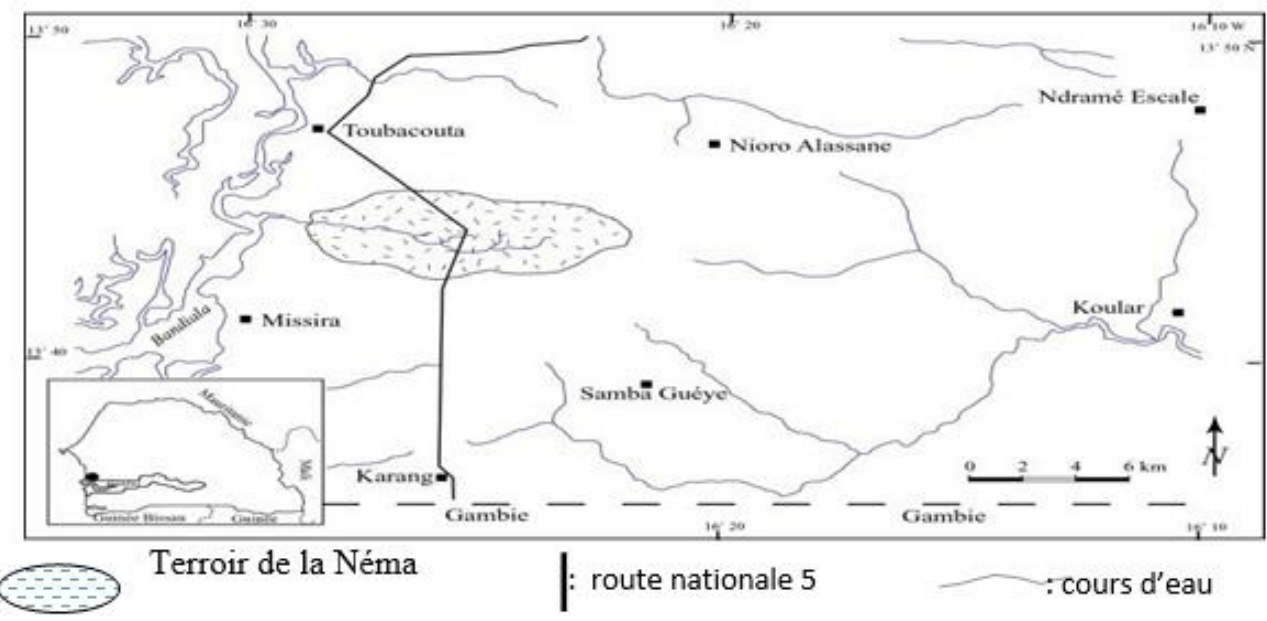

Figure 2. Carte de situation du terroir de la Néma (source : Coly et al., 2001)

Situé dans la région naturelle du Sine (Fatick), ce terroir appartient au vaste bassin sédimentaire côtier sénégalo-mauritanien (Michel, 1969). Le bassin de la Néma est drainé par une rivière de même nom, orientée Est/ Ouest, et qui se jette dans le Bandiala, un bras de mer des îles du Saloum.

Les sols sablo-argileux, correspondent aux sols ferrugineux tropicaux lessivés (SCET, 1966).

Le climat est tropical semi-aride, de type soudano-sahélien. Les températures moyennes extrêmes sont $36,3^{\circ} \mathrm{C}$ (en avril, le mois le plus chaud) et $26,2^{\circ} \mathrm{C}$ (en décembre, le mois le plus frais). La pluviométrie moyenne annuelle (1980-2013) est de 572,5 mm avec un coefficient de variation de 29\%. La saison des pluies s'étale en moyenne sur 126 jours (mai à octobre). Le mois d'août est le plus pluvieux avec $311 \mathrm{~mm}$ (Coly, 1999). 


\section{Méthodes utilisées}

\section{Collecte des données}

Un suivi régulier des différents groupes d'exploitations (grandes exploitations, exploitations moyennes et petites exploitations) définis par Coly et al. (2011) a été effectué. Pour ce faire, une fiche de suivi a été établie. Ainsi, au cours d'un entretien avec les chefs d'exploitations, les questions abordées ont trait au ramassage, au transport et à l'épandage du fumier, aux contraintes d'utilisation, à la quantité appliquée par ha, aux rendements obtenus dans les champs fumés, aux spéculations bénéficiaires du fumier, etc.

Pour l'évaluation des rendements en fane et en gousses d'arachide dans les champs fumés et non fumés (témoins), un échantillon de trois parcellles fumées et trois parcelles non fumées a été retenu par groupe d'exploitations. La méthode des carrés de rendement a ensuite été utilisée dans chaque parcelle. Cette méthode vise à évaluer la quantité de fane et de gousses à partir des placettes carrées de $2 \mathrm{~m}$ de côté. Pour ce faire, tous les plants d'arachide de la placette sont récoltés et les gousses séparées des fanes. Au total, 20 placettes carrées ont été utilisées par champ. Des échantillons de fanes et de gousses sont prélevés, pesés et mis en sachets et ramenés au laboratoire. Ils sont ensuite mis à sécher à l'étuve à $60^{\circ} \mathrm{C}$ jusqu'à poids constant.

\section{Analyse chimique du fumier}

Des échantillons de fumier ont été prélevés dans des exploitations suivies. Ils ont été analysés au laboratoire commun de Chimie de l'Institut de Recherche pour le Développement (IRD) au Sénégal selon les techniques classiques d'analyse des éléments minéraux contenus dans les végétaux. Le fumier utilisé est obtenu par les méthodes décrites par Coly et al. (2013).

Le bilan établi a reposé essentiellement sur les données de production de gousses et de fanes, la quantité de fumier apportée et la fixation symbiotique de l'azote.

Les éléments nutritifs sont importés par le fumier, la fixation de l'azote atmosphérique par les légumineuses alors que les exportations se font par les productions principales (graines) et les productions secondaires (résidus de récolte).

\section{Traitement des données}

Les données collectées lors du suivi des exploitations ont été traitées à l'aide du tableur excel du logiciel XLSTAT.

L'évaluation de la quantité de fane d'arachide et de gousses produite en poids sec est réalisée à partir d'une constante $\mathrm{K}$ (teneur de matière sèche dans $100 \mathrm{~g}$ de matière fraîche, \%MS).

$$
\mathrm{P}_{\mathrm{kgMS}}=\mathrm{P}_{\mathrm{kgPF}} \mathrm{x} \text { K } \% \mathrm{MS}
$$


Le poids frais (PF) moyen est obtenu à partir de la moyenne du poids des échantillons prélevés dans les champs suivis. Le poids sec $(\mathrm{P})$ moyen est obtenu à partir de ces mêmes échantillons après passage à l'étuve jusqu'à obtention d'un poids constant.

La constante $\mathrm{K}$ a été calculée pour les différents groupes d'exploitations définis.

Pour chaque groupe, le poids sec moyen de fanes produit par ha est calculé avec la formule suivante :

$$
\left.\mathrm{Ps}=\left(\left(\sum \mathrm{i}=1 \text { à n pi } * \mathrm{Kx}\right) / \mathrm{n}\right) / 4\right) * 10000=\left(\left(\sum \mathrm{i}=1 \text { à n pi } * \mathrm{~K}\right) / \mathrm{n}\right) * 2500
$$

Ps = poids sec de fane produite à l'hectare $;$ pi = poids frais de fane par carré de $4 \mathrm{~m}^{2} ; \mathrm{K}=$ teneur de matière sèche dans $100 \mathrm{~g}$ de matière fraîche pour le groupe $\mathrm{x} ; \mathrm{n}=$ nombre d'échantillons (carrés délimités dans les champs) dans l'ensemble des exploitations du groupe considéré.

La production de fane dans les groupes d'exploitations a été calculée sur la base du rendement par ha. Ainsi :

$\mathrm{P}=\mathrm{R} * \mathrm{SE}$ (où $\mathrm{P}=$ Production $; \mathrm{R}=$ Rendement et $\mathrm{SE}=$ Superficies emblavées).

La même formule a été utilisée pour les deux produits (fanes et gousses).

Le bilan des éléments nutritifs (N, P, K) a été fait sur la base de leur importation et exportation au niveau des champs fumés.

\section{Exportations des éléments nutritifs ( $\mathrm{N}$ Y K) au champ}

L'évaluation des quantités d'éléments exportés a été faite. Ainsi, les quantités d'Azote $(\mathrm{N})$ exportées sont calculées par la formule suivante : Qté N-export.ara. = $($ fane-ara-prod.* ten. N-fane ara $)+($ gousses-ara-prod.* ten. N-gousses-ara) Où

- Qté N- export.ara = quantité d'azote exportée des champs d'arachide. -fane-ara- prod. =quantité de fane d'arachide produite $(\mathrm{kg})$. -ten.N-fane ara. =teneur en azote $(\mathrm{N})$ de la fane d'arachide $(\mathrm{g} / \mathrm{kg})$. -gousses-ara-prod. = quantité de gousses d'arachide produite $(\mathrm{kg})$. -ten. $\mathrm{N}$-gousses-ara $=$ teneur en azote des gousses d'arachide $(\mathrm{g} / \mathrm{kg})$.

\section{Importations des éléments nutritifs}

Les quantités d'azote apportées dans les champs fumés sont calculées par les formules suivantes :

Qté N-fum. ara -imp = qté-fum.app.*ten-N-fum*coef.eff. N-fum*(1-fen-p) où

-qté N-fum-ara -imp = Quantité d'azote importé dans les champs d'arachide par le fumier ;

-qté-fum.app.= quantité de fumier apportée $(\mathrm{kg})$;

-ten-N-fum =teneur en azote du fumier $(\mathrm{g} / \mathrm{kg})$; 
-coef. eff. N-fum = coefficient d'efficacité de l'azote du fumier ;

-f-en-p $=$ fraction d'engrais perdue.

Cette formule est aussi appliquée pour le phosphore et le potassium.

$>\quad$ Qté $\mathrm{N}$-fix-ara $=(\mathrm{N}$-fane ara. $+\mathrm{N}$-gousses ara. $) * f-f i x *$ coef. eff.- $\mathrm{N}$ fum* $(1-f-e n-p)$ où

-qté $\mathrm{N}$-fix-ara. $=$ quantité d'azote atmosphérique fixée par les plantes d'arachide ;

-N-fane ara. = quantité d'azote contenue dans la fane produite ;

-N-gousses ara $=$ quantité d'azote contenue dans les gousses d'arachide produite;

-f-fix = fraction d'azote fixée.

Cette dernière formule ne concerne que l'azote atmosphérique, qui peut être fixée de façon symbiotique par les légumineuses.

En somme, qté N-ara-imp. = qté N-fum-ara-imp. + qté N-fix-ara. Où

Qté N-ara-imp. = quantité d'azote importée dans les champs d'arachide.

Différents coefficients ont été déterminés :

- la capacité de fixation de l'azote atmosphérique par les légumineuses : 0,75 (Camara, 1996) ;

- le coefficient d'efficacité des éléments nutritifs d'origine organique, lequel indique la fraction qui peut être effectivement utilisée par les plantes comparativement à ceux des engrais chimique. Ce coefficient est de 0,$6 ; 1,2$ et 1 respectivement pour l'Azote, le Phosphore et le Potassium.

Il permet aussi de donner une valeur aux éléments nutritifs d'origine organique, sachant leur efficacité par rapport à ceux de l'engrais chimique ;

- la fraction d'engrais perdue détermine le coefficient d'utilisation par les plantes, c'est-à-dire, la fraction effectivement disponible pour leur alimentation. Le niveau et les causes de pertes varient selon les différents types d'éléments nutritifs. Ainsi pour l'azote, les pertes sont principalement causées par la dénitrification, la volatilisation et la lixiviation alors que pour le Phosphore, elles sont surtout causées par immobilisation et pour le potassium, par lessivage. La fraction d'engrais perdue a été estimée à 0,4 pour l'azote et le Potassium et 0,5 pour le Phosphore (Camara, 1996).

\section{Résultats}

\section{Choix des parcelles à fumer et transport du fumier produit}

Les principales spéculations qui font l'objet d'une fertilisation avec le fumier sont le mil et l'arachide. Elles sont d'ailleurs les deux spéculations les plus pratiquées dans le terroir de la Néma.

Le fumier produit dans le terroir de la Néma est destiné à la fois aux champs de brousse et aux champs de case $(41,48 \%$ des exploitations), aux 
champs de case seulement (21,5\% des exploitations) et aux champs de brousse seulement (20\% des exploitations). En fait, le choix des parcelles devant recevoir le fumier n'est pas lié à la proximité à son lieu de production. Les paysans épandent le fumier dans les parcelles en baisse de fertilité quelque soit la distance.

Tableau 1. Choix des parcelles bénéficiaires du fumier

\begin{tabular}{cccc}
\hline $\begin{array}{c}\text { Types de } \\
\text { parcelles }\end{array}$ & $\begin{array}{c}\text { Champs de } \\
\text { case }\end{array}$ & $\begin{array}{c}\text { Champs de } \\
\text { brousse }\end{array}$ & $\begin{array}{c}\text { Champs de case et } \\
\text { de brousse }\end{array}$ \\
\hline $\begin{array}{c}\text { Nombre } \\
\text { d'exploitations }\end{array}$ & 29 & 27 & 56 \\
\hline$(\%)$ & 21,5 & 20 & 41,48 \\
\hline
\end{tabular}

Le transport du fumier vers les parcelles se fait avec des charrettes pour l'essentiel des exploitants. En effet, 77\% des exploitants interrogés affirment avoir transporté leur fumier à l'aide d'une charrette et seulement 2,22\% soutiennent l'avoir transporté à la tête. Les résultats du suivi des exploitations montrent que les grandes exploitations disposent en moyenne de 2 charrettes par exploitation et celles des autres groupes possèdent en moyenne 1 charrette par exploitation, ce qui rend possible l'épandage du fumier dans les parcelles quelque soit leur distance.

\section{Épandage du fumier dans les champs}

Le fumier est produit au sein de l'exploitation à partir des déjections des animaux en stabulation (essentiellement animaux de trait) qui se mélangent avec les fanes d'arachide servies aux animaux durant toute la saison sèche. L'utilisation après décomposition complète de ce mélange (fumier) pour la fertilisation des champs est une pratique assez répandue dans le terroir de la Néma.

La dose de fumier appliquée a varié d'un groupe à l'autre. : 158,4 kg/ha dans le groupe des petites exploitations à $1173,3 \mathrm{~kg} / \mathrm{ha}$ dans le groupe des grandes exploitations. L'épandage du fumier est généralisé dans ce dernier groupe $(100 \%)$ et faiblement pratiquée dans le groupe des petites exploitations $(9,1 \%)$. En terme de nombre de charrettes de fumier épandu par an, on a 20 ; 7 et 0,36 respectivement pour les groupes des grandes exploitations, des exploitations moyennes et des petites exploitations.

Ces résultats s'expliqueraient par l'importance numérique du cheptel dans les grandes exploitations (en moyenne 8 bovins extensifs, 2 bovins de trait, 1 asin, 2 équins, 2 ovins, 1 caprin par exploitation) et la faiblesse de l'effectivité du cheptel dans les exploitations de petite taille (en moyenne 1 bovin extensif, 2 bovins de trait, 1 asin et 1 caprin par exploitation). Les faibles quantités de fumier appliquées dans les petites exploitations seraient donc liées à un déficit de production de celui-ci. 
La principale contrainte liée à l'épandage du fumier est, selon les exploitants agricoles, l'insuffisance ou parfois l'inexistence de'un moyen de transport adéquat (charrette) du fumier, ce qui fait que l'éloignement de certaines parcelles (champs de brousse) pose réellement problème. A cette contrainte s'ajoute la faible capacité de production des exploitations en fumier. En effet, la quantité de fumier produite est largement en deçà des besoins. Ceci s'expliquerait soit par l'insuffisance de litière apportée (résidus de récolte) dans le lieu de stabulation des animaux soit par l'absence d'animaux en stabulation dans les exploitations (coly et al., 2013).

\section{Effet du fumier sur le rendement en fanes et en gousses fane d'arachide}

L'examen de la Figure 2 permet de voir que le fumier a amélioré le rendement en fane d'arachide. Le rendement varie en effet de $1162 \mathrm{~kg} / \mathrm{ha}$ (champs témoins) à $805 \mathrm{~kg} / \mathrm{ha}$ (champs fumés) pour le groupe des grandes exploitations, de 919,7 dans les champs témoins à $1415,68 \mathrm{~kg} / \mathrm{ha}$ dans les champs fumés (exploitations de taille moyenne) et enfin de $628,6 \mathrm{~kg} / \mathrm{ha}$ dans les champs témoins à 1 160,3 kg/ha dans les champs fumés (exploitations de petite taille).

Dans le terroir, les rendements moyens varient de 1230,3 kg/ha pour les champs fumés à 903,4 kg/ha pour les champs témoins.

Excepté le groupe des grandes exploitations, quelque soit le groupe d'exploitations considéré, le rendement est toujours plus élevé avec la fumure organique.

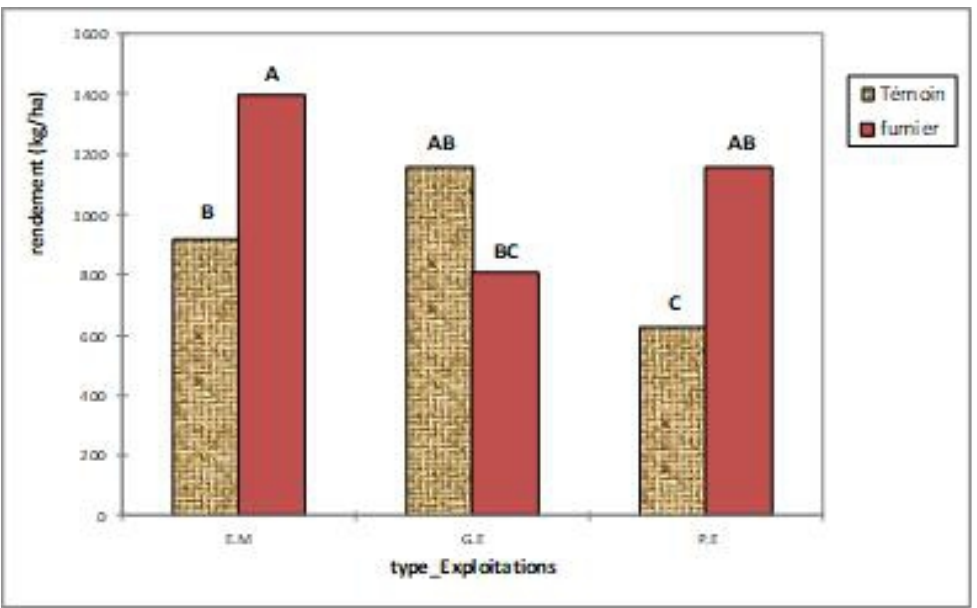

Figure 2. Effet du fumier sur les rendements (kg-MS/ha) en fanes d'arachide selon les groupes d'exploitations du terroir.

$\mathrm{G} . \mathrm{E}=$ grandes exploitations ; E.M= exploitations moyennes ; P.E = petites exploitations

Effet $=R_{f}-R_{s f}\left(R_{f}=\right.$ rendement avec fumier $; R_{s f}=$ rendement sans fumier ou témoin) 
Les rendements de fanes induits par l'application de fumier sont donc plus élevés dans les groupes des exploitations moyennes et des petites exploitations. Les différences observées par rapport aux parcelles témoins sont hautement significatives $(\mathrm{p}=0,001)$ dans les petites exploitations et très hautement significatives $(\mathrm{p}=0,0001)$ dans les moyennes selon l'ANOVA et le test de comparaison de moyennes de Newman et Keuls appliqué au seuil de $5 \%$. Cependant, dans les grandes exploitations, la différence des rendements obtenus entre les champs fumés et témoins est non significative $(p=0,053)$.

\section{Les gousses}

Le graphique ci-dessous montre globalement un effet fumier sur le rendement en gousses dans le terroir. L'effet est plus marqué dans les moyennes et petites exploitations. Les rendements sont passés de 279,3 (champs témoins) à $412,38 \mathrm{~kg} / \mathrm{ha}$ (champs fumés) dans les premières et de 146,67 (champs témoins) à $289 \mathrm{~kg} / \mathrm{ha}$ (champs fumés) dans les secondes.

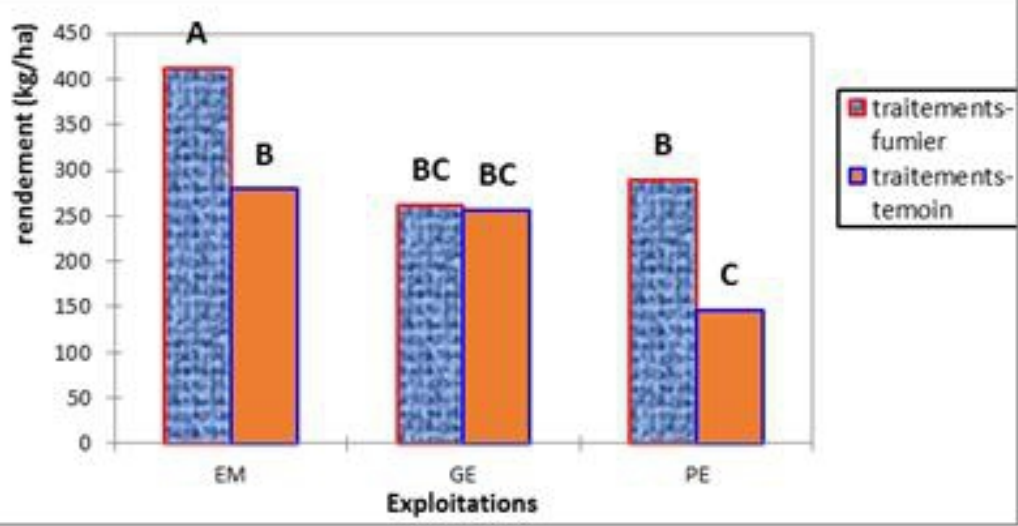

Figure 3. Effet du fumier sur les rendements (kg-MS/ha) en gousses d'arachide selon les groupes d'exploitations du terroir de la Néma.

L'ANOVA et le test de comparaison de moyennes de Newman et Keuls appliqué au seuil de 5\% révèlent, entre les champs témoins et les champs fumés, une différence de rendement hautement significative dans les exploitations moyennes $(\mathrm{p}=0,003)$, et significative dans les petites exploitations $(\mathrm{p}=0,037)$. Toutefois, cette différence dans les grandes exploitations n'est pas significative $(p=0,057)$.

\section{Bilan des éléments nutritifs dans les champs d'arachide fumes}

Le tableau 2 présente les importations, exportations et flux net des éléments NPK dans les champs d'arachide fumés suivant les groupes d'exploitations agricoles. 
Tableau 2. Bilan des nutriments (NPK) au champ suivant les groupes d'exploitations (kg/ha).

\begin{tabular}{|l|c|c|c|c|c|}
\cline { 2 - 6 } \multicolumn{1}{c|}{} & \multicolumn{1}{c|}{$\begin{array}{c}\text { Grandes } \\
\text { exploitations }\end{array}$} & $\begin{array}{r}\text { Exploitations } \\
\text { moyennes }\end{array}$ & $\begin{array}{r}\text { Petites } \\
\text { exploitations }\end{array}$ & Moyenne & Ecart type \\
\cline { 2 - 6 } & \multicolumn{5}{c|}{ Azote } \\
\hline Importations & 8,70 & 13,72 & 9,50 & 10,64 & 2,69 \\
\hline Exportations & 15,86 & 48,63 & 34,91 & 33,13 & 16,45 \\
\hline & $-7,16$ & $-34,91$ & $-25,41$ & $-22,49$ & 14,10 \\
\hline Importations & 1,30 & 0,8 & 0,10 & 0,73 & 0,60 \\
\hline Exportations & 1,86 & 3,27 & 2,41 & 2,51 & 0,71 \\
\hline Flux net & $-0,56$ & $-2,47$ & $-2,31$ & $-1,78$ & 1,06 \\
\hline & \multicolumn{5}{|c|}{ Phosphore } \\
\hline Importations & 0,8 & 0,7 & 0,2 & 0,57 & 0,32 \\
\hline Exportations & 6,693 & 11,74 & 8,596 & 9,01 & 2,55 \\
\hline Flux net & $-5,89$ & $-11,04$ & $-8,40$ & $-8,44$ & 2,58 \\
\hline
\end{tabular}

Les importations d'azote dans les champs d'arachide fumés varient de 8,7 (Grandes exploitations) à 13,72 kg (Exploitations moyennes) par ha, soit en moyenne 10,64 kg/ha à l'échelle du terroir. La variabilité est assez élevée ; en effet le coefficient de variation est de $25,28 \%$. Quant aux exportations, elles sont aussi plus élevées dans les exploitations moyennes $(48,63 \mathrm{~kg} / \mathrm{ha})$ et plus faibles dans les grandes exploitations $(15,86 \mathrm{~kg} / \mathrm{ha})$; la variabilité y est aussi grande : 49,65\%. Les exportations sont donc nettement plus élevées que les importations.

Ainsi le flux net, qui traduit la différence entre importations et exportations, est partout déficitaire. Le déficit atteint $7,16 \mathrm{~kg} / \mathrm{ha}$ dans le groupe des grandes exploitations pour les valeurs les plus faibles et $34,91 \mathrm{~kg} / \mathrm{ha}$ dans le groupe des exploitations moyennes pour les valeurs les plus élevées. L'écart type associé au flux net moyen est de $14,10 \mathrm{~kg} / \mathrm{ha}$, soit $62,7 \%$; cela traduit une forte variabilité entre les différents groupes d'exploitations.

Les importations du Phosphore sont de très faibles $(1,3 \mathrm{~kg} / \mathrm{ha})$ à insignifiantes $(0,1 \mathrm{~kg} / \mathrm{ha})$, tandis que les exportations varient de 1,86 à 3,27 $\mathrm{kg} / \mathrm{ha}$, soit en moyenne $2,51 \mathrm{~kg} / \mathrm{ha}$ à l'échelle du terroir. Le bilan est partout déficitaire. Ce déficit, comme pour l'azote, est plus marqué en valeurs absolues dans le groupe des exploitations moyennes $(2,47 \mathrm{~kg} / \mathrm{ha})$ et plus faible dans le groupe des grandes exploitations $(0,56 \mathrm{~kg} / \mathrm{ha})$. Dans ce dernier groupe, le bilan paraît équilibré.

Contrairement à l'azote, les importations de phosphore sont plus élevées dans le groupe des grandes exploitations (1,3 kg/ha). Elles sont plus faibles dans le groupe des petites exploitations $(0,1 \mathrm{~kg})$. Par rapport aux importations moyennes pour l'ensemble du terroir $(0,73 \mathrm{~kg} / \mathrm{ha})$, la variabilité est assez importante (écart type de 0,60 kg/ha). Les exportations de Phosphore dans les champs fumés sont plus élevées dans le groupe des exploitations moyennes $(3,27 \mathrm{~kg} / \mathrm{ha})$ et plus faible dans le groupe des grandes exploitations 
$(1,86 \mathrm{~kg} / \mathrm{ha})$ avec une moyenne de $2,51 \mathrm{~kg} / \mathrm{ha}$ dans le terroir.

Le déficit moyen dans le terroir est relativement faible avec $1,78 \mathrm{~kg} / \mathrm{ha}$, avec un coefficient de variation relativement élevé $(59,55 \%)$; la différence entre les groupes d'exploitations paraît ainsi grande.

En ce qui concerne le bilan en Potassium des sols des champs d'arachide fumés, le flux net est partout déficitaire. Ce déficit est plus important dans le groupe des exploitations moyennes $(11,04 \mathrm{~kg} / \mathrm{ha})$ et plus faible dans le groupe des grandes exploitations $(5,89 \mathrm{~kg} / \mathrm{ha})$. Le déficit moyen à l'échelle du terroir est de $8,44 \mathrm{~kg} / \mathrm{ha}$, avec un coefficient de variation de $30,57 \%$.

Les importations de Potassium dans le terroir sont en moyenne de 0,57 $\mathrm{kg} / \mathrm{ha}$. Elles sont plus élevées dans le groupe des grandes exploitations $(0,8$ $\mathrm{kg} / \mathrm{ha})$ et plus faibles dans le groupe des petites exploitations $(0,2 \mathrm{~kg} / \mathrm{ha})$. La variabilité du flux net est aussi élevée : $2,58 \mathrm{~kg} / \mathrm{ha}$, soit $30,56 \%$. Les exportations de Potassium sont plus importantes dans le groupe des exploitations moyennes $(11,74 \mathrm{~kg} / \mathrm{ha})$ et plus faibles dans le groupe des grandes exploitations $(6,69 \mathrm{~kg} / \mathrm{ha})$, avec un écart type de $2,55 \mathrm{~kg} / \mathrm{ha}$, soit $28,3 \%$; c'est le coefficient de variation le plus faible enregistré dans les champs d'arachide fumés.

\section{Discussion}

Ce travail s'est intéressé d'une part aux conditions d'utilisation du fumier et d'autre part à l'effet de son épandage sur le bilan des éléments nutritifs et sur les rendements agricoles dans le terroir de la Néma.

Des groupes d'exploitations agricoles ont été définis sur la base de leurs caractéristiques socio-économiques (Coly et al., 2011), ceci dans le but de bien cerner des différentes modalités de gestion du fumier suivant les caractéristiques des exploitations.

Les résultats obtenus montrent que l'épandage du fumier est une pratique très commune dans le terroir de la Néma. Si dans le groupe des exploitations de grande taille cette pratique est systématique, dans celui des petites exploitations elle est quelque peu limitée par l'insuffisance ou parfois l'inexistence de moyen de transport approprié mais aussi par l'insuffisance de la quantité de fumier produite.

La dose de fumier appliquée varie selon la taille de l'exploitation de $158,4 \mathrm{~kg} /$ ha à $1173,3 \mathrm{~kg} / \mathrm{ha}$. Ces doses sont très faibles, comparées à celles avancées par Moutaharou et al. (2002) à la suite d'une étude effectuée au Bénin. Leurs résultats indiquent une dose variant de 5 à 11 tonnes de fumier à l'hectare dans leur zone d'étude. Les facteurs limitantes à la production de fumier dans les exploitations pourraient être la faiblesse de la taille du cheptel mis à contribution pour la production de fumier mais aussi l'insuffisance de la litière apportée dans le lieu de stabulation des animaux (coly et al., 2013). Il 
conviendrait ainsi, pour pallier le déficit de litière et donc de fumier dans l'exploitation, d'apporter en plus des refus de fanes d'arachide, des pailles de céréales, ce qui permettra au-delà de la fane d'arachide de valoriser la biomasse végétale non consommable par le bétail. Cette litière incorporée aux fèces permettrait une amélioration quantitative et qualitative du fumier produit dans l'exploitation (Dugué, 1998).

D’une manière générale, les parcelles qui bénéficient des amendements organiques sont celles qui présentent des signes de baisse de fertilité quelque soit la distance. Ce résultat s'oppose à celui obtenu au Benin par Moutaharou et al. (2002). Selon ces auteurs, les parcelles qui font l'objet de fertilisation organique sont celles qui sont proches du lieu de production du fumier (habitat). Les cultures qui bénéficient de l'épandage du fumier sont principalement le mil et l'arachide avec une priorité accordée à la culture vivrière (mil). Ces deux spéculations sont pratiquées suivant un cycle bisannuel dans le terroir.

Le transport du fumier de son lieu de production vers les parcelles se fait d'une manière générale à la charrette. Seule une très faible proportion des exploitants le fait à la tête du fait du manque de moyen de transport.

La production de gousses et de fane d'arachide dans les champs fumés et dans les champs témoins fait apparaître globalement dans le terroir un effet positif du fumier sur les rendements. Il apparaît ainsi que l'on peut améliorer la fertilité des sols par la restitution organique, et donc améliorer la production, ce qui corrobore les observations de Berger (1996) et Nguo Balingene et al. (2016). Le gain induit par l'épandage du fumier est plus important dans le groupe des exploitations moyennes $(+495,98 \mathrm{~kg} /$ ha pour la fane et $+133,08$ $\mathrm{kg} / \mathrm{ha}$ pour les gousses). Les faibles gains observés dans les autres groupes pourraient être liés aux précédents culturaux ou à la non maîtrise de la culture.

Les principales contraintes liées à l'épandage du fumier dans le terroir sont l'insuffisance des moyens de transport du fumier vers les champs et la faiblesse des quantités de fumier produits dans l'exploitation,ce qui se traduit par des doses de fumier appliquées très faibles comparées aux valeurs obtenues dans la sous-région comme au Benin où nous avons des doses à l'hectare qui varient de 5 à 11 tonnes.

Les diverses productions réalisées au champ induisent des flux de nutriments dont l'importance dépend du niveau d'intensification (fumier) et donc du mode de gestion des résidus de récolte mais aussi de l'importance des productions agricoles.

Le bilan de l'azote est déficitaire dans les champs fumés. Ce déficit est particulièrement marqué dans le groupe des exploitations moyennes. La fane et les gousses d'arachide exportent plus d'azote qu'on en apporte par le biais du fumier. Ce déficit s'expliquerait d'une part par les faibles teneurs en azote du fumier $(2,15 \mathrm{~g} / \mathrm{kg})$ et d'autre part par les faibles quantités de fumier 
apportées à l'hectare (coly et al., 2013). Malgré ce déficit en azote, les rendements apparaissent globalement sensiblement améliorés par rapport aux parcelles témoins. L'existence d'effet synergique de la matière organique et les éléments minéraux contenus dans le fumier (Maltas et al., 2012) pourraient expliquer les bons rendements observés. Cela laisse présager de réelles perspectives pour l'utilisation du fumier qui favorise un enrichissement progressif du sol en matière organique (Maltas et al., 2011).

Compte tenu des modes d'utilisations de la fane d'arachide dans le terroir de la Néma (alimentation du bétail et vente), une bonne partie des éléments nutritifs reviendra, après recyclage de la fane dans les lieux de stabulation, vers les champs par l'intermédiaire du fumier (Coly, 2003). Dans ces conditions toutefois, les pertes sont de $55 \%$ pour N, $50 \%$ pour P et $35 \%$ pour K contenus dans la fane (Camara, 1996). Les éléments nutritifs contenus dans la fane vendue constituent une perte totale car ils ne reviendront jamais au champ, ce qui est préjudiciable pour la fertilité des sols.

Le déficit en azote dans les champs fumés, plus important dans le groupe des exploitations moyennes $(34,91 \mathrm{~kg} / \mathrm{ha})$, est plus lié à l'importance de la production de fane observée à ce niveau qu'à la faiblesse des quantités de fumier répandue par hectare car le groupes des petites exploitations apportent une quantité de fumier beaucoup plus faible à l'hectare.

Le bilan de $\mathrm{P}$ dans les champs d'arachide fumés, globalement négatif dans le terroir $(-1,78 \mathrm{~kg} / \mathrm{ha})$, n'est pas loin de l'équilibre dans les grandes exploitations $(-0,56 \mathrm{~kg} / \mathrm{ha})$. Cela s'expliquerait alors par les quantités de fumier apportées par hectare qui apparaissent plus élevées mais aussi par les faibles rendements de fane dans les champs fumés $(805 \mathrm{~kg} / \mathrm{ha})$ par rapport aux autres groupes.

Nous avons noté aussi dans le terroir de la Néma un déficit permanent relativement important en $\mathrm{N}$ alors que le bilan phosphoré est parfois équilibré, ce qui représente un corollaire des prélèvements d'azote par les plantes plus important que celui de $\mathrm{P}$ (Camara, 1996). Ces résultats sont concordants avec ceux de Van der Pol (1990), lesquels ont montré en zone soudanienne au MaliSud que le bilan phosphoré est équilibré, tandis que le bilan azoté des terres est déficitaire. Le déficit en $\mathrm{N}$ atteint dans le terroir de la Néma 22,5 Kg/ha contre, $25 \mathrm{~kg} / \mathrm{ha}$ dans la zone du Mali-Sud.

Globalement, le bilan en $\mathrm{K}$ est négatif dans le terroir. Le déficit en Potassium dans les champs d'arachide fumés apparaît plus important dans le groupe des exploitations moyennes, ce qui laisse apparaître un lien entre l'importance de la production en fane et le bilan en éléments nutritifs. En effet c'est le groupe où le rendement le plus important en fane d'arachide dans les champs fumés est observé.

Ce bilan ne prend pas en compte le stock initial d'éléments nutritifs du sol.Aussi les apports dus aux dépôts atmosphériques et à la sédimentation ont 
été négligés de même que les exportations liées au lessivage et à l'érosion.

\section{Conclusion}

Il apparaît à travers les résultats de ce travail que dans le terroir de la Néma l'épandage du fumier dans les parcelles est une pratique très commune.

Le choix des parcelles devant recevoir le fumier n'est pas déterminé par la proximité de la parcelle à son lieu de production mais plutôt lié au niveau de fertilité de la parcelle. Le fumier produit dans le terroir est destiné principalement aux cultures du mil et de l'arachide.

Le moyen de transport du fumier le plus courant est la charrette bien que son acquisition ne soit pas généralisée particulièrement dans les petites exploitations dont les moyens sont assez limités. Ceci constitue une contrainte à l'épandage du fumier en plus de la modicité des quantités de fumier produit par rapport aux besoins des exploitations.

Il en résulte une faiblesse des doses de fumier appliquées à l'hectare qui ,malgré tout, permettent d'améliorer sensiblement les rendements en fane et en gousses d'arachide dans le terroir.

Le bilan en azote, phosphore et potassium est déficitaire dans les champs d'arachide fumés à l' échelle du terroir, et ceci, du fait de la faiblesse des doses de fumier apportées à l'ha et de sa faible teneur en éléments nutritifs.

Il apparait ainsi opportun de poursuivre les investigations en tenant compte du stock initial en éléments nutritifs du sol, ceci en vue de mieux cerner l'effet des exportations des cultures sur le sol et de mieux apprécier les quantités d'éléments nécessaires à une bonne restauration de la fertilité du sol.

\section{References:}

1. Akpo, L. E., Coly, I., Sarr, D., Ngom, D., \& Ndao, S. (2002). Impact des modes d'utilisation des terres sur la diversité floristique dans le bassin de la Néma en zone soudano-sahélienne (Sine- Saloum, Sénégal) : $5^{\text {ème }}$ Conférence inter-régionale sur l'environnement et l'eau. Actes de colloque, Ouagadougou, SOGIF, 635p.

2. Akpo, L. E., Coly, I., Sarr, D., Ngom, D., \& Ndao, S. (2003). Modes d'occupation des terres et gestion des ressources forestières en zone soudannienne de l'Afrique de l'Ouest : l'exemple du bassin versant de la Néma au Sénégal. Actes de colloque, Montpellier, 1-8.

3. Akpo, L. E., Coly, I., Sarr, D., Ngom, D., \& Ndao, S. (2004). Modes d'utilisation des terres et diversité floristique dans le terroir de la Néma en zone Semi-aride (Sénégal, Afrique de l'Ouest), Journal of agriculture and environnement for international development, vol. 98, No. 3/4 P 165-180.

4. Berger, M. (1996). L'amélioration de la fumure organique en Afrique soudano-sahélienne. Agriculture et Développement numéro hors série. 
5. Borderon, A. (1989). Rapport d'évaluation du projet Mali-Sud III Tome 1.

6. Bossissi, N. (2010). Détermination du choix de fertilisants d'une rotation maaïs-haricot volubile en fonction de la capacité des ménages, Mémoire, UCB, $72 \mathrm{p}$.

7. Camara, O. S. (1996). Utilisation des résidus de récolte et du fumier dans le cercle de Koutiala : bilan des éléments nutritifs et analyse économique. Exploitation optimale des éléments nutritifs en élevage, $120 \mathrm{p}$.

8. CMDT (1993). Zonage Mali-Sud, Développement et gestion des ressources. Région de Koutiala, Projet gestion terroir San-Koutiala 98 p.

9. Coly, I. (1999). Caractérisation des ressources ligneuses du terroir de la Néma dans le Niombato (Saloum) au Sénégal. DEA FST/UCAD, Dakar, $38 \mathrm{p}$.

10. Coly, I., Akpo, L. E., Dacosta, H., Diome, F., \& Malou, R. (2001). Caractérisation agro-écologique du terroir de la Néma au Saloum (Sénégal): les systèmes d'utilisation des terres et diversité du peuplement ligneux, Journal des Sciences vol. $1 \mathrm{~N}^{\circ} 2$, 2001 p.8-18.

11. Coly, I. (2003). Utilisation des résidus de récolte dans un terroir soudano-sahélien : disponibilité, bilan des éléments nutritifs et analyse économique dans le terroir de la Néma au Sénégal. Doct. de $3^{\text {ème }}$ cycle Biol. Vég. FST/UCAD, Dakar. 75 p.

12. Coly, I., Diome, F., Dacaosta, H., Malou, R., \& Akpo, L. E. (2011). Typologie des exploitations agropastorales du terroir de la Néma (Sénégal, West Africa). International Journal of Biological and Chemical Sciences 5 (5) : 1941-1959.

13. Coly, I., Diop, B., \& Akpo, L. E. (2013). Transformation locale des résidus de récolte en fumier de ferme dans le terroir de la Néma au Saloum (Sénégal), Journal of Applied Biosciences 70: 5640-5651.

14. Dugué, P. (1998). Flux de biomasse et gestion de la fertilité à l'échelle des terroirs. Etude de cas au Nord Cameroun et essai de généralisation aux zones de savane. Doc. CIRAD- TERA n ${ }^{\circ} 29 / 98,68$ p.

15. Kaasschieter, G. A. \& Coulibaly, Y. (1995). Rentabilité de l'utilisation de de niébé (Vigna unguiculata) comme supplément avec la paille de mil (Pennisetum typhoides) par des taurillons. Rapport Production Soudano-Sahélienne $N^{\circ}$. Exploitation optimale des éléments nutritifs en élevage, $29 \mathrm{p}$.

16. Kleene, P., Sanogo, B., \& Vierstra, G. B. (1989). Présentation, objectifs et méthodologie du volet deFonsebougou (1977-1987). Collection : Systèmes de productions Rurale au Mali : vol. 1. 
17. Liénou, G. (1995). Relations écoulements de surfaces - écoulements souterrains dans le bassin versant de Néma (Sine-saloum, Sénégal). DEA Université Cheikh Anta Diop de Dakar.

18. Madjikam, P. S. (2000). Bilan fourrager disponible dans un terroir agro-pastoral : importance des résidus de récolte dans le terroir de la Néma au Niombato (Centre-Sénégal). Doc. Vét., Dakar, 9, 54 p.

19. Maltas, A., Charles, R., \& Sinaj, S. (2011). Fertilité du sol et productivité des cultures: effet des apports organique et du labour. Recherche Agronomiques Suisse 2 (3), 120-127.

20. Maltas, A., Charles, R., Bovet, V., \& Sinaj, S. (2012). Effet à long terme des engrais organiques sur le rendement et la fertilisation azotée des cultures. Recherche Agronomique Suisse 3 (3): 156-163.

21. Michel, P. (1969). Les bassins des fleuves Sénégal et Gambie. Etude géomorphologique. Thèse de Doctotat es sc. sTRASBOURG : $161 \mathrm{p}$.

22. Moutaharou, A., Djenontin, J. A., \& Wennink, B. (2002). Valorisation des résidus de récolte dans l'exploitation agricole au Nord Benin : utilisation du fumier produit dans le parc de stabulation des bœufs. Savanes Africaines: des espaces en mutation, des acteurs face aux nouveaux defis. Actes de colloque, Maroua, Cameroun. 8p. $\mathrm{N}^{\circ} 2$ : 5-19.

23. Ndao, S. (2001). Pratiques agricoles et gestion et gestion de l'arbre dans les parcs agroforesters de la Néma en zone soudano-sahélienne (Sine-saloum, Sénégal). DESS, CRESA, Niamey, $70 \mathrm{p}$.

24. Ngom, D. (2000). Importance de l'arbre dans les systèmes de production traditionnels du terroir de la Néma dans le Niombato (Saloum, Sénégal). DESS, CRESA, Niamey, 72 p.

25. Nguo Balingene, P., Monde, G., \& Seburiri, T. (2016). Effet des différents fertilisants organiques sur la culture de l'aubergine à Saka (R. D. Congo) Annales de l'UNIGOM, vol. VI, N², 111-120.

26. SCET (1966). Aménagement des vallées du bas Saloum et du sine Pakala. Etude pédologique : vallée de la Néma. Société centrale pour l'équipement du terroir coopération, $26 \mathrm{p}$.

27. Van der Pol (1990). Soil mining. An unseen contributor to farm income in southern Mali. KIT Bulletin, 325, KIT, Amsterdam. 